\title{
Value of Broncho-Alveolar Lavage in Diagnosis of Newly Developed Lung Infiltrates in Mechanically Ventilated Patients
}

\author{
Yasser Mostafa ${ }^{1}$, Nevine Abd Elfattah ${ }^{1}$, Rehab M. Mohammed ${ }^{1}$, Lobna Roshdy ${ }^{2}$, Mahmoud Ibrahim ${ }^{2}$ \\ ${ }^{1}$ Chest Department, Faculty of Medicine, Ain Shams University, ${ }^{2}$ Chest Department, Egypt Air Hospital \\ Corresponding author: Mahmoud Ibrahim, Email: drmoudheet2@gmail.com
}

\begin{abstract}
Background and Objectives: in a developing country, cost effectiveness is an important consideration. The aim of this study was to investigate the efficiency and safety of mini broncho-alveolar lavage (mini BAL) samples in the diagnosis of newly developed lung infiltrates in mechanically ventilated patients.

Methods: fifty mechanically ventilated patients with newly developed lung infiltrates were assessed by miniBAL and subsequent microbiological examination. An infant Ryle catheter FG-10 was used as the inner catheter, a Nelaton catheter size 18 FG was used as the outer protective catheter and was blocked by sterile KY gel instead of the pre-packaged catheters.

Results: eighty six percent of samples collected by Mini-BAL technique from mechanically ventilated patients showed positive growth for culture and sensitivity. Thirty percent of patients had bi microbial infection while fourteen had polymicrobial infection. Fungal infection (Candida) was the most frequent isolated pathogen (32\%) followed by Gram-negative bacteria (Klebsiella) (30\%). Legionella was the commonest isolated atypical bacteria.

Conclusion: this study highlighted the mini-BAL technique as a simple, safe, cheap, available and noninvasive bedside procedure for acquiring uncontaminated lower respiratory secretions in patients with newly developed pulmonary infiltrates.
\end{abstract}

Key words: Broncho-Alveolar Lavage, Newly Developed Lung Infiltrates, Mechanically Ventilated Patients.

\section{INTRODUCTION}

The complexity of patients in the intensive care unit (ICU), together with the recent advances in radiographic images have led to new perspectives in the use of chest X-ray in the ICU. The American College of Radiology Consensus Committee recommends and maintains that chest $\mathrm{x}$-ray in patients with cardiopulmonary disease or those receiving mechanical ventilation should be performed daily ${ }^{(\mathbf{1})}$.

Nosocomial pneumonia, a common ICU infection, affects $27 \%$ of all critically ill patients, where $86 \%$ of it is associated with mechanical ventilation ${ }^{(2)}$. According to the infectious diseases society of America / American Thoracic Society (IDSA/ATS) (2) hospital acquired/nosocomial pneumonia (HAP) is pneumonia that occurs $48 \mathrm{~h}$ or more after admission and did not appear to be incubating at time of admission. On the other hand, VAP is a type of HAP that develops more than 48-72 $h$ after endotracheal intubation ${ }^{(3)}$. Moreover, VAP occurs in $28 \%$ of patients who receive mechanical ventilation, where its rate of occurrence varies with the duration of mechanical ventilation. Estimated rates are $3 \%$ per day for the first 5 days, $2 \%$ per day for days $6-10$, and $1 \%$ per day after day $10{ }^{(4)}$. The diagnostic clinical Triad for VAP consists of pulmonary infection signs including fever, purulent secretions, and leucocytosis, together with bacteriologic evidence of pulmonary infection, and radiological suggestion of pulmonary infection ${ }^{(5)}$.
There are multiple ways to get sputum samples from intubated patients such as tracheal aspirate, blind protected brushes and blind mini lavage (blind miniBAL). Patients that have endotracheal tubes can undergo procedures besides BAL for diagnosis of VAP. Both blind protected brushes and blind minilavage [blind mini-BAL] can be done in these patients. These procedures are less expensive and more readily available. The results obtained from these procedures have also been compared to lung histology and cultures from patients with VAP, and the data from blind mini-BAL has a sensitivity and specificity of $80 \%$ when correlated with the sensitivity of the bronchoscopic BAL ${ }^{(6)}$.

This study aimed to investigate the efficiency and safety of mini broncho-alveolar lavage (mini BAL) samples in the diagnosis of newly developed lung infiltrates in mechanically ventilated patients.

\section{PATIENTS AND METHODS}

The current study included 50 mechanically ventilated patients of different ages and gender admitted to Respiratory Intensive Care Unit at Egypt Air Hospital suffering from respiratory failure. The study period was two years. The study was approved by the Ethics Board of Ain Shams University.

The enrolled patients were on mechanical ventilation for more than 48 hours in whom chest x-ray showed newly developed pulmonary 
infiltrates and ventilator associated pneumonia was clinically suspected.

VAP was suspected when a new infiltrate or the progression of a prior stable infiltrate appeared on chest radiograph starting 72 hours or more after admission or mechanical ventilation. Moreover, at least two of the following signs of pneumonia were also required: temperature $>38{ }^{\circ} \mathrm{C}$ or $<36{ }^{\circ} \mathrm{C}$, leucocytosis $>12,000 / \mathrm{mm} 3$ and sputum production; increase amount and change colour to yellow, greenish or pus and microbiological criteria (positive culture obtained by mini-BAL catheter with colony count $\geq 10^{3}$ as a cut off value for positive culture).

The exclusion criteria: included mechanical ventilation < 48 hours, tracheal aspirates less than one $\mathrm{ml}$, presence of lung shadows or pneumonia before mechanical ventilation.

Patients were subjected to full clinical examination and laboratory investigations

\section{Follow up for developing lower respiratory tract infection by:}

Clinical assessment: thorough daily assessment of modified clinical pulmonary infection score (CPIS).

\section{Radiological assessment}

Microbiological evaluation: by quantitative culture of respiratory secretion using mini-BAL manoeuvre to collect samples from lower respiratory tract. This was done after 48 hours of mechanical ventilation.

\section{Procedure:}

A Nelaton catheter size (I8-FG), in which distal end was cut just above its lateral eyes, was used as an outer protective catheter, an infant Nelaton catheter size (10-FG) was used as the inner catheter and sterile K-Y gel was used to block the distal end of the outer catheter. Sterile gloves and 3 syringes $20 \mathrm{ml}$ each of normal saline and a specimen container. Prior to the procedure. The FIO2 was set at 1.0. using sterile gloves. The distal end of Nelaton catheter was plugged with tiny piece of sterile, K-Y gel to prevent contamination by bronchial secretions of inner aspect of catheter during advancement. The Nelaton catheter, which serves as an outer catheter was gently advanced into the artificial airway until resistance is met, indicating the catheter is wedged into the distal airway then retracted $4-5 \mathrm{~cm}$. The infant Nelaton catheter, which serves as an inner catheter is advanced in a telescopic manner through the outer catheter, extruding the K-Y gel plug. A $20 \mathrm{ml}$ syringe was connected to the inner catheter to administer its content of normal saline that was aspirated again using the same $20 \mathrm{ml}$ syringe while maintaining the catheter position and aspiration process was repeated as necessary (up to $60 \mathrm{ml}$ ) until an appropriate specimen is obtained. The sample was poured into sterile container carefully to avoid contamination with closure of the lid tightly. Both catheters were removed together from the airways. The FIO2 was reduced back to the original setting ${ }^{(7)}$.

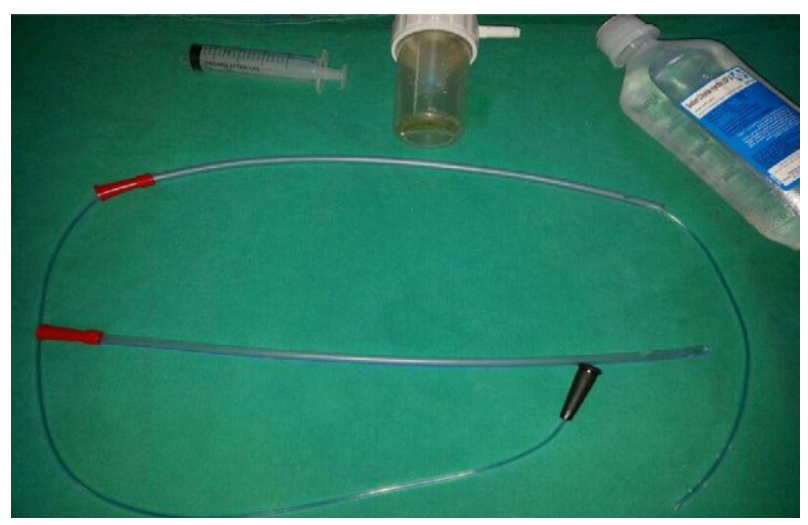

Figure (1): Mini-BAL equipments.

Microbiological examination of the mini-BAL fluid:

Direct film: Gram stain: for the presence of polymorph-nuclear leucocytes and for detection of microorganisms by their staining characters, zeil-Nelsen stain: for microscopic examination of acid fast bacilli, giemsa stain: specifically for toxoplasma, pneumocystis carinii and for the presence of fungus spores and / or hyphae.

\section{Isolation of different microorganisms:}

By routine culture media: A $0.01 \mathrm{ml}$ sterile calibrated loop was placed into the respective specimen and then onto the center of three media plates (blood agar, chocolate agar, and macconkey agar). The isolated pathogens were identified by their morphological and biochemical reactions as catalase, coagulase and oxidase tests. Stained films from bacterial growth were examined by microscopy for the type of bacteria, Gram reaction (Gram-positive or Gram-negative) and morphology of the bacteria (cocci, diplococcic, rods or coccobacilli). 
Colony count: Bacterial culture growth was quantitated according to the number of colonies observed per plate. It was counted as follows: < 10 colonies per plate represented < $10^{3} \mathrm{cfu} / \mathrm{ml}, 10$ to 100 colonies per plate represented $10^{3}$ to $10^{4} \mathrm{cfu} / \mathrm{ml}, 100$ to 1000 colonies per plate represented $10^{4}$ to $10^{5} \mathrm{cfu} / \mathrm{ml}$ and > 1000 colonies per plate represented > $10^{5} \mathrm{cfu} / \mathrm{ml}$. All identified microorganisms were reported with their antibiotic sensitivities. Colony count $\geq 10^{3} \mathrm{cfu} / \mathrm{ml}$ was taken as cut off value for positive culture according to JosepMaria et al. ${ }^{(8)}$ and Herve et al. ${ }^{(9)}$.

CHROM agar Orientation: CHROM agar Orientation can differentiate mixed flora easily without the need of subcultures. Antimicrobial susceptibility tests can be performed directly from primary isolates.

Plate Reading: E coli $\rightarrow$ dark pink to reddish, Enterococcus $\rightarrow$ turquoise blue, Proteus $\rightarrow$ brown halo, Klebsiella, Enterobacter and Serratia $\rightarrow$ metallic blue, S.aureus $\rightarrow$ golden, opaque and small, Citrobacter $\rightarrow$ metallic blue with red halo, S.saprophyticus $\rightarrow$ pink, opaque and small, Candida albicans $\rightarrow$ colourless, Streptococcus agalactiae $\rightarrow$ Light blue and Pseudomonas aeruginosa $\rightarrow$ Translucent and cream to blue.

Legionella Culture: The standard medium used is buffered charcoal yeast extract (BCYE) agar, which provides iron and L-cysteine, with or without antibiotics, essentials for the growth of Legionella.

Chlamydia Procedure: The Quick Chlamydia Test is a lateral flow immunoassay intended for the rapid, qualitative detection of chlamydia directly from specimens.

\section{CHROM agar Candida:}

Interpretation: Microorganism Typical colony appearance; C.albicans $\rightarrow$ green, C.tropicalis $\rightarrow$ metallic blue, C.krusei $\rightarrow$ pink and fuzzy, C.kefyr and C.glabrata $\rightarrow$ mauve-brown and other species $\rightarrow$ white to mauve.

\section{Statistical analysis}

Data were collected, tabled and statistically analyzed using SPSS vs. 15.

\section{RESULTS}

Table (1): Demographic characteristics, comorbidities, pulmonary diseases, duration of mechanical ventilation and $\mathrm{Pao} 2 / \mathrm{FIo} 2$ ratio

\begin{tabular}{|l|c|}
\hline Gender & $\mathrm{N}(\%)$ \\
Male & $31(62)$ \\
Female & $19(38)$ \\
\hline Age years & \\
Min-max & $49-77$ \\
mean \pm SD & $64.66 \pm 5.5$ \\
\hline Co-morbidity & $\mathrm{N}(\%)$ \\
DM & $13(26)$ \\
Hypertension & $8(16)$ \\
CVD (IHD, CHF, ICM) & $15(30)$ \\
CVS & $14(28)$ \\
RI & $5(10)$ \\
Extra pulmonary malignancy & $7(14)$ \\
\hline Pulmonary & \\
COPD & $\mathrm{N}(\%)$ \\
ILD & $13(26)$ \\
Pulmonary malignancy (lung mass, mesothelioma, & $2(4)$ \\
metastases) & $7(14)$ \\
OHV & $1(2)$ \\
CAP & $3(6)$ \\
\hline Duration of MV(days) & \\
Min-max & $4-12$ \\
mean \pm SD & $7.32 \pm 1.75$ \\
\hline PaO2/FiO2 & $151-250$ \\
Min-max & $212.64 \pm$ \\
Mean \pm SD & 29.37 \\
ARDS N (\%) (PaO2/FiO2 <200) & $23(46)$ \\
\hline
\end{tabular}

The present study included 50 mechanically ventilated patients with mean age \pm SD was $64.66 \pm 5.5$ years old. $62 \%$ of them were males and $38 \%$ were females. The mean duration \pm (SD) of mechanical ventilation was $7.32 \pm 1.75$ days and the mean Pao2/FIo2 ratio was (212.64 \pm 29.37) with $46 \%$ of enrolled patients having Pao2/FIo2 ratio $<200$ (acute respiratory distress syndrome (ARDS)).

Table (2): The number of isolated pathogens among the included patients.

\begin{tabular}{|l|c|}
\hline Isolated pathogen/patient $(\mathbf{n}=\mathbf{5 0})$ & $\mathbf{N}(\boldsymbol{\%})$ \\
\hline None & $7(14 \%)$ \\
Unimicrobial & $21(42 \%)$ \\
Bimicrobial & $15(30 \%)$ \\
Polymicrobial & $7(14 \%)$ \\
\hline
\end{tabular}

Table (3): Frequency of isolated organisms (microbial yield).

\begin{tabular}{|l|c|}
\hline \multicolumn{1}{|c|}{ Pathogen } & N $(\%)$ \\
\hline Legionella & $10(20)$ \\
Candida & $16(32)$ \\
Chlamydia & $6(12)$ \\
Acenitobacter & $4(8)$ \\
M. tuberculosis & $1(2)$ \\
Anaerobes & $2(4)$ \\
Staph aures & $6(12)$ \\
Klebsiella & $15(30)$ \\
MRSA & $3(6)$ \\
Pseudomonas & $9(18)$ \\
\hline
\end{tabular}


Moreover, in the current study, the microbial cultures were positive for $43(86 \%)$ patients; $42 \%$ of them had uni-microbial isolates in their cultures while $15 \%$ had bi microbial isolates and $14 \%$ had polymicrobial isolates in their cultures. Gram-negative organisms were the most frequent isolated organisms $(56 \%) .30 \%$ of them were Klebsiella, $18 \%$ were Psudomonas and $8 \%$ were Acintobacter. Fungal infection candida was 32\%. Atypical bacteria Legionella was $20 \%$ and Chlamydia was $12 \%$. Grampositive organisms: methicillin sensitive Staphylococcus aureus (MSSA) was $12 \%$ and methicillin resistant Staphylococcus aureus (MRSA) was $6 \%$. Anaerobes were (4\%). Tuberculosis was $2 \%$.

Table (4): The frequency of multi-drug resistant (MDR) organisms.

\begin{tabular}{|l|c|}
\hline \multicolumn{1}{|c|}{ MDR(n=11) } & N (\%) \\
\hline Acinetobacter & $4(8)$ \\
Klebsiella & $3(6)$ \\
MRSA & $3(6)$ \\
Pseudomonas & $1(2)$ \\
\hline
\end{tabular}

Among the isolated pathogens: all isolated Acinetobacter $(8 \%)$ were multi-drug resistant (MDR), along with $6 \%$ of isolated Klebsiella and $2 \%$ of isolated organisms Pseudomonas.

Table (5): The correlation between CPIS and microbiolog.ical yield among the included patients.

\begin{tabular}{|c|c|c|c|c|c|c|c|}
\hline \multirow{2}{*}{1} & \multicolumn{2}{|c|}{$\begin{array}{c}\text { Negative } \\
\text { microbiology (n=7) }\end{array}$} & \multicolumn{2}{|c|}{$\begin{array}{c}\text { Positive } \\
\text { microbiology (n=43) }\end{array}$} & \multirow{2}{*}{$\begin{array}{c}\text { Test } \\
\text { value }\end{array}$} & \multirow{2}{*}{ P-value } & Sig. \\
\cline { 2 - 6 } & No. & $\%$ & No. & $\%$ & & & \\
\hline CPIS $<6$ & 5 & $71.4 \%$ & 11 & $25.6 \%$ & 5.815 & 0.016 & S \\
\hline CPIS $>6$ & 2 & $28.6 \%$ & 32 & $74.4 \%$ & & \\
\hline
\end{tabular}

There was a statistically significant positive correlation between the clinical pulmonary infection score (CPIS) and microbiological yield among the studied patients $(\mathrm{p}<0.05)$.

Table (6): Correlation between number of isolated pathogens per patient and the development of ARDS.

\begin{tabular}{|c|c|c|c|}
\hline Pathogens/patient & $\operatorname{ARDS}(n=23)$ & $\mathrm{X} 2$ & $\mathrm{p}$ \\
\hline None $(\mathrm{n}=7)$ & $1(14.3)$ & \multirow{4}{*}{7.9} & \multirow{4}{*}{0.04} \\
\hline Unimicrobial $(\mathrm{n}=21)$ & $12(57.4)$ & & \\
\hline Bimicrobial $(n=15)$ & $9(60)$ & & \\
\hline Polymicrobial $(n=7)$ & $1(14.3)$ & & \\
\hline
\end{tabular}

There was a statistically significant correlation between number of isolated organisms per patient and the development of ARDS ( $p<0.05$ ).

\section{DISCUSSION}

The diagnostic Blind mini-BAL is a less expensive, minimally invasive and more readily available. Bedsides, it is gaining increasing recognition as an alternative diagnostic procedure to acquire uncontaminated lower respiratory secretions in patients with suspected VAP with reported sensitivity and specificity of $80 \%{ }^{(\mathbf{1 0})}$.

The cut off value in this was chosen after Josep-Maria et al. ${ }^{(8)}$. Also, Herve et al. ${ }^{(9)}$ study who compared 4 sampling methods; blind tracheal aspirate, blind protected telescopic catheter (miniBAL technique) and bronchoscopic protected catheter for the diagnosis of VAP. It showed that the best threshold for blind protected telescopic catheter (mini BAL) was between $10^{2}$ and $10^{3} \mathrm{cfu} /$ $\mathrm{ml}$. The microbiological criteria of the present study matched with Josep-Maria et al. ${ }^{(10)}$ who compared the quantitative culture with the microscopical examination of intracellular organisms of mini-BAL samples for diagnoses of VAP and used positive quantitative cultures of the samples obtained by mini-BAL $>10^{3} \mathrm{cfu} / \mathrm{ml}$.

In addition, the current study matched with Herve et al. ${ }^{(9)}$ study who compared 4 sampling methods; blind tracheal aspirate, blind protected telescopic catheter (mini-BAL technique) and bronchoscopic protected catheter for the diagnosis of VAP and had shown that the best threshold for blind protected telescopic catheter (mini BAL) was between $10^{2}$ and $10^{3} \mathrm{cfu} / \mathrm{ml}$.

The microbiological criteria were not matching with that of Nseir et al. ${ }^{(11)}$ as they used the cut off value for positive tracheal aspirate quantitative culture $\geq 10^{6} \mathrm{cfu} / \mathrm{ml}$, and for BAL sample $\geq 10^{4}$. The present study also not agree with Craven et al. ${ }^{(12)}$ who used cut off value tracheal aspirate with positive quantitative culture $\geq 10^{5} \mathrm{cfu} / \mathrm{ml}$ or BAL sample $\geq 10^{4} \mathrm{cfu} / \mathrm{ml}$ for diagnosis of VAT. The disagreement between the present study and the previous studies was because they used unprotected tracheal aspirate samples while in the present study protected mini-BAL samples were used. So, lower colony count was considered positive. The microbiological criteria also did not match with that used by Montgomery et al. ${ }^{(13)}$ who used Gram stain of tracheal aspirate for diagnosis of VAT and VAP while in the present study quantitative culture of protected mini-BAL sample was used with cut off value $<10^{3} \mathrm{cfu} / \mathrm{ml}$ for positive culture.

In the current study, the microbial tests showed that Gram-negative organisms are the most 
frequent isolated pathogens, (30\%) of them were Klebsiella and Legionella were 20\%, Pseudomonas was $18 \%$, Acinetobacter was $8 \%$. Gram-positive organisms were $12 \%$, methicillin sensitive Staphylococcus aureus (MSSA) was 6\%, methicillin resistant Staphylococcus aureus (MRSA) was 6\%. Atypical bacteria Chlamydia was $12 \%$. Fungal infection candida was $32 \%$. Anaerobe was $4 \%$. Tuberculosis was $2 \%$. If Gram's stain didn't reveal any bacteria, and no bacterial growth was retrieved by culture, this may suggest infection with Legionella, viruses, fungi or anaerobic bacteria. This result was in agreement with Marik and Careau ${ }^{(14)}$ who drew the attention toward the possibility of these agents as causative organisms of VAP, and highlighted that clinicians should take into account such microorganisms and consider them during empirical therapy.

Our results demonstrated that fungal infection candida was $32 \%$. These results were close to those of many investigators who recently recognized Candida spp. colonization as a risk factor for systemic candidias and bacterial infections. They added that isolation of Candida species (spp.) from respiratory tract secretions is common, but the diagnosis of Candida pneumonia is rare. Garnacho-Montero et al. ${ }^{(15)}$ reported that in ICU patients, evidence pints the fact that Candida tracheobronchial colonization increased the risk for subsequent Pseudomonas aeruginosa (P. aeruginosa) ventilator-associated pneumonia (VAP) and other multidrug resistant bacteria. They explained that inflammation elicited by Candida colonization might affect the immune defence, which in turn favours bacterial development and they recommended that antifungal therapy could potentially reduce the risk for P. aeruginosa VAP. They emphasized on the message that Candida spp. should no longer be seen as a simple bystander and might constitute possible leads for new therapies to treat pneumonia.

Our results were close to Abd-Elfattah ${ }^{(16)}$ who found that the most prevalent organism in his study was Klebsiella pneumonia (42.5\%). Our results were close to Heyland et al. ${ }^{(17)}$ who found that the most common organisms cultured from tracheal aspirates, of patients with VAP, were Klebsiella pneumoniae, Acinetobacter species, Pseudomonas aeruginosa, Methicillin resistant Staphylococcus aureus (MRSA) Methicillin sensitive Staphylococcus aureus (MSSA). On the other hand, Laura et al. (18) found that the commonest organism isolated from 100 patients with VAP was Pseudomonas aeruginosa (29.0\%), Staphylococcus aureus (28.0\%), Klebsiella (19.0\%) and Acinetobacter spp. (18.0\%).

The results of our study agreed with the results of Mokhless et al. ${ }^{(19)}$ they revealed that Candida was the commonest organism isolated accounting for $23.3 \%$, while $16 \%$ only were of significant count $\left(\geq 10^{5} \mathrm{CFU} / \mathrm{ml}\right)$. This was followed by Pseudomonas aeruginosa $21.6 \%$ and $15 \%$ were of significant count, then the polymicrobial growth $20 \%$ and $16 \%$ with significant count, Staphylococcus aureus $16 \%$ and $10 \%$ with significant count.

The significantly high rate of Gramnegative bacilli in our study and many other studies probably indicated the high incidence of prolonged hospital stay and the prolonged duration of mechanical ventilation that predisposed the patients to acquire infections from the multidrug-resistant pathogens. In contrast, other authors reported other bacterial strains as Acinetobacter baumanii and Streptococcus ${ }^{(20)}$. Noteworthy, airway intubation is associated with increased frequency of Gramnegative bacterial colonization of the upper and lower respiratory tracts, followed by rapid growth of these Gram-negative bacteria and pneumonia (43). This reflects their ability to survive in the hospital environment ${ }^{\text {(21). }}$.

Consequently, prior use of antibiotics significantly decreased the incidence of VAP caused by Gram-positive cocci or $\mathrm{H}$. influenzae, but significantly increased the rate of VAP caused by $P$. aeruginosa ${ }^{(22)}$. Noteworthy, the etiological agents of VAP may differ according to patients, units, hospitals or countries. The main epidemiological patterns might not only vary from unit to unit, but also in a given unit over the course of time and this is true for their associated susceptibility patterns. Thus, reported differences could frequently be explained by local specificities (22).

The results of our study agreed with the results of Hassan et al. ${ }^{(23)}$ who reported the detection of legionella and Chlamydia pneumonia in VAP cases while no cases were positive for Mycoplasma pneumoniae. Thus, empirical antimicrobial regimens should cover Chlamydia and Mycoplasma. Furthermore, El-Ebiary et al. ${ }^{(24)}$ also diagnosed six 
cases of Legionella pneumonia among patients with definite VAP. Using specific culture for Legionella and serology for Legionella pneumophila, Mycoplasma pneumoniae Coxiella burnetti, and Chlamydia pneumonia. Only Legionella was diagnosed in 2 patients by serology and in 4 patients by culture. Our results draw attention towards the possibility of these rarely diagnosed agents as being not infrequent causative agents for VAP. The prevalence of such atypical pathogens must be taken into consideration while tailoring the empiric antimicrobial coverage of patients diagnosed with VAP.

Our cultures revealed that $30 \%$ of the patients had bimicrobial and $14 \%$ of the patients had polymicrobial infection. These results matches those of Joseph et al. (25) who reported polymicrobial infection in $27.8 \%$ of VAP patients. Moreover, only $13.46 \%$ of cultures were polymicrobial. This may be because of exclusion of patients with ARDS, cavitary lung disease, lung cancer, as well as tuberculosis patients, and patients with congenital or acquired immunodeficiency.

In the current study, there was a highly statistically significant relation between the method of collection of the samples and the prevalence of the organisms as $86 \%$ of samples collected by Mini-BAL technique from mechanically ventilated patients showed positive growth for culture and sensitivity. This matched the reported data from the study conducted by Abd-Elfattah ${ }^{(16)}$ who found that sensitivity of Min-BAL in the diagnosis of VAP reached $100 \%$ when correlated with the sensitivity of the bronchoscopic BAL.

These results were in agreement with the data including mini-BAL where the concordance percentage with BAL reported in an overview of 7 different studies on blinded sampling techniques (73 to $100 \%$. Complications of BAL were more frequent and carried a higher risk as it required sometimes reintubation with a larger ET to allow the FOB. Two complications were observed with the new min-BAL, but were transitory and benign. In the literature ${ }^{(17)}$, most of the authors recommended the use of blind protected techniques over the BAL as it had less complications rendering it less invasive and safer. In the present study, the relation between number of the isolated organisms and ARDS showed that there was statistically significant correlation. Pneumonia-related ARDS can be caused by bacterial, viral, fungal, and even parasitic pathogens. The differences in the types and percentages of organisms isolated between the current study and the above mentioned studies may be due to the wide spectrum of etiologic agents which can cause VAP which vary by hospital, type of ICU and patients. So, local surveillance of microbiological data is important.

\section{CONCLUSION}

This study highlighted that the new miniBAL proved to be a simple, safe, cheap, available and non-invasive bedside procedure for acquiring uncontaminated lower respiratory secretions in patients with newly developed pulmonary infiltrates and suspected VAP.

\section{REFERENCES}

1. Henschke CI, Yankelevitz DF, Wand A, Davis SD, Shiau M (1997): Chest radiography in the ICU.Clin Imaging, 21(2):90-103.

2. American Thoracic Society-Infectious Diseases Society of America (2005): Guidelines for the management of adults with hospital-acquired, ventilator-associated, and health care-associated pneumonia. Am J Respir Crit Care Med., 171: 388-416.

3. Kalil AC, Metersky ML, Klompas M et al. (2016): Management of adults with hospital acquired and ventilator-associated pneumonia: 2016 clinical practice guidelines by the infectious diseases society of America and the American thoracic society. Clin Infect Dis., 63:e61.

4. Baker AM, JW Meredith, and EF Haponik (1996): Pneumonia in intubated trauma patients. Microbiology and outcomes. Am. J. Respir. Crit. Care Med., 153:343-349.

5. Fàbregas N, Ewig S, Torres A, El-Ebiary M, Ramirez J, de La Bellacasa JP et al. (1999): Clinical diagnosis of ventilator associated pneumonia revisited: comparative validation using immediate post-mortem lung biopsies. Thorax., 54(10):867-73.

6. Bregeon F, Papazian L and Visconti A (2000): Diagnostic accuracy of protected catheter sampling in ventilator- associated bacterial pneumonia. Eur Respir J., 16(5): 969-975.

7. Abd Elfattah N, Madkour A and Sharkawy S (2009): The Efficiency of a New, Cheap and Safe Method in Acquiring a Mini-BAL Sample for VAP Diagnosis: An Initial Egyptian Trial. Egyp J Chest Dis Tuber., 55(2): 56-60. 
8. Josep-Maria Sirvent, Vidaur L, Gonzalez S, Castro P, de Batlle J, Castro A, Bonet A. (2003): Microscopic Examination of Intracellular Organisms in Protected Bronchoalveolar Mini Lavage Fluid for the Diagnosis of +Ventilator- Associated Pneumonia. Chest., 123:518-523.

9. Herve Mentec, May-Michelangeli L, Rabbat A, Varon E, Le Turdu F, Bleichner G (2004): Blind and bronch- oscopic sampling methods in suspected ventilator associated pneumonia. Intensive Care Med., 30(7): 1319-1326.

10. .Bregeon F, Papazian L and Visconti A (2000): Diagnostic accuracy of protected catheter sampling in ventilator- associated bacterial pneumonia. Eur Respir J., 16(5): 969-975.

11. .Nseir S, Favory R, Jozefowicz E et al. (2008): Antimicrobial treatment for ventilator- associated tracheobronchitis: a randomized, controlled, multicenter study. Crit Care, 9:238-245.

12. Craven DE, Lei $Y$, Ruthazer $R$ et al. (2013): Incidence and outcome of ventilatorassociated tracheobronchitis and pneumonia. Am J Med., 126:542-549.

13. Montgomery AB, Vallance S, Abuan T, Tservistas M, Davies A (2014): A randomized double-blind placebo-controlled dose-escalation phase 1 study of aerosolized amikacin and fosfomycin delivered via the PARI investigational eFlow ${ }^{\circledR}$ inline nebulizer system in mechanically ventilated patients. J Aerosol Med Pulm Drug Deliv., 27(6):441-8.

14. Marik PE, Careau P (1999): The role of anaerobes in patients with ventilator associated pneumonia and aspiration pneumonia. Chest, 115:178-183.

15. Garnacho-Montero J, Olaechea $P$, AlvarezLerma $\mathbf{F}$, Alvarez-Rocha $\mathbf{L}$, Blanquer $\mathbf{J}$, Galván B, Rodriguez A, Zaragoza R, Aguado JM, Mensa J, Solé A, Barberán J (2013): Epidemiology, diagnosis and treatment of fungal respiratory infections in the critically ill patient. Rev Esp Quimioter., 26(2):173-88.

16. Abdel-Fattah MM (2005): Surveillance of nosocomial infections at a Saudi Arabian military hospital for a one-year period. Ger Med Sci., 3: Doc06.
17. Heyland D, Cook D, Dodek P et al. (2006): A Randomized Trial of Diagnostic Techniques for Ventilator-Associated Pneumonia. N Engl J Med., 355:2619-30.

18. Laura d, Carolina $P$ and Renata $C$ (2008): Ventilator-Associated Pneumonia in an Adult Clinical-Surgical Intensive Care Unit of a Brazilian University Hospital: Incidence, Risk Factors, Etiology, and Antibiotic Resistance. The Brazilian Journal of Infectious Diseases, 12(1):80-85.

19. Mokhless NAS, El-Mofty MF, Hanafi NF, Fayed AM, Asser SL (2010): Atypical bacteria in ventilator associated pneumonia; an Egyptian university hospital experience, Research in Medical Microbiology and Immunology and Critical Care Medicine, Faculty of Medicine, Alexandria University, Journal of American Science, 6(12): 1074- 1079.

20. Chastre J, Fagon JY (2002): Ventilator associated pneumonia. Am J Respir Crit Care Med., 165: 867-903.

21. Dominic RS, Prashanth HV, Shenoy S, Baliga $S$ (2012): A clinico- microbiological study of ventilator-associated pneumonia in a tertiary care hospital. International Journal of Biological and Medical Research, 3(2):1651-1654.

22. Rello J, Ausina V, Ricart M, Castella J, Prats G (1993): Impact of previous antimicrobial therapy on the etiology and outcome of ventilator-associated pneumonia, Chest, 104: 1230- 1235.

23. Hassan A, Samy A, Bedewy K (2007): VAP: the role of typical bacteria and atypical bacteria and Fungi. Comparison between endotracheal aspirates and bronchoalveolar lavage, EJB., 1(1): 53-61.

24. El-Ebiary M, Torres A, González J et al. (1993): Quantitative cultures of endotracheal aspirates for the diagnosis of ventilatorassociated pneumonia. Am J Respir Crit Care Med., 147:1552-1557.

25. Joseph NM, Sistla S, Dutta TK, Badhe AS, Rasitha D, Parija SC (2009): Ventilatorassociated pneumonia in a tertiary care hospital in India: Incidence and risk factors. Journal of Infection in Developing Countries, 3: 771-777. 